\title{
Can Mobile Game Development Foster Student Interest in Computer Science?
}

\author{
Stan Kurkovsky \\ Department of Computer Science \\ Central Connecticut State University \\ New Britain, CT, USA \\ kurkovskysta@ccsu.edu
}

\begin{abstract}
Mobile culture refers to an increasingly important role that mobile communication and mobile media play in our everyday lives. Young people are often viewed as the driving force behind the innovation in mobile technology, since they comprise the majority of early adopters and most avid users of mobile gadgets and applications, especially mobile games. Many contemporary college students grew up surrounded by computer games and electronic gadgets and, therefore, may better relate to mobile technology than to the desktops dominating current academic environment. This paper makes the case for using mobile game development as a motivational tool and a learning context in computing curriculum, which is supported by the results of a large student survey. The paper also presents a case study illustrating how mobile game development can be successfully integrated into a breadth-first introductory Computer Science course.
\end{abstract}

Keywords-mobile games; mobile culture; curriculum; student learning; motivation

\section{INTRODUCTION}

The term "mobile culture" has emerged to reflect a phenomenal role that mobile devices play in our everyday lives. Growing importance of mobility and anytime anywhere access to information services has created a brand new set of shared practices, relationships, social norms, and rituals [9]. Mobile phones, as enablers of anytime anywhere access to instant communication, have extended their capabilities by incorporating various forms of media and, as a consequence, their social significance has dramatically increased.

Most current college students (approximately 18-25 years of age) have grown up surrounded by mobile gadgets and immersed in mobile culture. Many young people in this age group would find it difficult to live a day without using their mobile phones to talk, check email, send text messages, and play games. There is a clearly evident convergence trend for mobile gadgets to combine the features of a media player, photo and video camera, navigation device, and, last but not least, a communication device. In the eyes of many current students, computing technology is associated with these small and ubiquitous devices instead of larger desktops that are commonly used today in the academic environment. We believe that there is an excellent opportunity to use this affinity of young people to mobile gadgets and turn it into a motivational tool that can stimulate their interest in Computer Science and help them become more engaged in their studies.

This paper presents the case for using mobile game development and mobile computing as a learning context and motivational tool to spark and foster student interest in Computer Science (CS). As CS educators, we should take advantage of the students' affinity to mobile culture. This paper discusses different aspects of the role that mobile devices and mobile media play in today's culture and their influence on young people comprising the population of typical college students. This discussion is supported by the results of an extensive survey conducted in November and December 2008, which studied behavioral patterns and opinions of several hundred college students from Connecticut. The paper reviews different strategies and challenges of integrating mobile computing and mobile game development into the computing curricula. The paper is concluded with a case study describing a breadth-first introductory CS course with a strong emphasis on mobile game development.

\section{CAn Mobile Computing Help?}

A complex combination of factors has been identified as the cause of current low enrollments in CS programs. Decreased appeal of computing as a field of study and/or a future career is probably of the highest significance. To make matters worse, many existing students choose to change their major after taking a few core CS courses because they perceive course material as dry and having no relevance to the real word applications [1]. It is ever more important for CS programs to remain relevant to the current reality in the eyes of our students and engage them by showing strong ties between computing and their everyday lives. We believe that by using mobile game development it is possible to make course material more relevant to students; this also may help making a stronger connection to the real-world applications and gadgets that surround our students.

In the eyes of modern college students, there is a significant gap in the way they use computing technology and how computers are used in academic environments. High school and college students, who are in the focus of current recruitment and retention efforts, are very likely to have had a mobile phone or a game console as their first computing device. In fact, current sales of mobile devices exceed those of PCs by 
over 20 to 1 . According to an IBM study released in October 2008 , over $50 \%$ of consumers in the US, UK and China would prefer to use their mobile devices over PCs for their Internet usage [11]. Most notably, the vast majority of those who intended to replace their PCs with mobile devices were 15-30 years old.

Positive perception of CS as a discipline is most often formed by a close role model, while students' perception formed solely by popular culture may not be so positive [8]. In order for CS programs to stay relevant to everyday lives of students, they must see that the academic model is evolving with the rest of the world. Students expect faculty to be knowledgeable about modern technology and fluent with current technical terminology. Actively using modern mobile devices as a part of the coursework may help portray the program and its faculty as more relevant and up to date. Since the vast majority of current students carry mobile phones with them at all times, being able to develop and immediately run an application on their phone may offer an experience that is as practical and as close to the real world as it gets.

\section{What About Mobile Culture?}

Mobile culture refers to an increasingly important role that mobile communication and mobile media play in our everyday lives ranging from personal interaction to social networking, from popular culture to politics. Special terms and words have emerged to refer to mobile phones, which demonstrates their prominent position in many cultures, especially those with higher rates of mobile technology saturation. Words referring to a mobile phone include keitai (something you carry with you) in Japanese and kännykkä (an extension of the hand) in Finnish.

Rich mobile communication has become an indispensable part in maintaining many social relationships and online social networks. In recent presidential elections in the US, online social networks were vital in helping people connect with one of their candidates who would actively update his profile on Facebook and post updates on Twitter with an enviable frequency. As a motivational tool, it was increasingly important for the candidate's supporters to have access to these tidbits in real time. Many researchers and analysts point out that such nearly real-time communications available anytime and anywhere help maintain the ties among members of many online social groups, not limited to that of a presidential candidate.

Mobile phones play an important role in the modern media culture. Wide availability of camera phones enables many eyewitnesses of breaking news to upload pictures and videos of events as they happen. Prominent examples range from London bombings in July 2005 to police shooting a young man in the back at a train station in California in January 2009. In many cases, live footage shot on a mobile phone by a bystander may become the only documental evidence of an important event, which is later broadcast on television, posted on the Internet, and discussed in blogs. On January 15, 2009, one the first and frequently used photographs of the US Airways flight 1549 crashing into Hudson River was taken using an iPhone and initially posted via Twitter.
Mobile interaction often has a more entertainment-oriented value and is incorporated into the structure of many television programs. For example, such TV shows as American Idol and Big Brother allow viewers to vote for contestants by sending text messages. TV show Deal or No Deal offers a Live TV Challenge where viewers can play along with the contestants in real time as the TV show airs. In both of these approaches, mobile phones provide a new feedback channel for viewers similar to calling in to a TV talk show. Additionally, mobile devices have become an extension of media distribution channels and broadcast platforms when users can download music and video to their devices. They have also become a facilitator of fan culture with users being able to download screens and ringtones helping them associate with a particular artist or popular culture phenomenon.

It is important to distinguish between two related concepts of mobile culture and mobile media. Mobile media is not a new concept. In fact, one might argue that newspapers and books are mobile media since they can be read anytime and anywhere. More versatile mobile media platforms would include different types of portable computing devices from laptops to smart and mobile phones. The strict technological boundary that once separated laptops from mobile phones may be vanishing, however. Starting in 2008 , there is a significant rise in popularity of small, light-weight and highly portable netbooks designed primarily for email and web-based applications. On the other end of the spectrum, there are "credible rumors" (as of June 2009) that Apple is set to release a large iPod-like tablet device with a 7"-9" screen suitable for comfortable web browsing, gaming, reading e-books, etc. However, it is their small form factor, along with features for consuming (web browsing, gaming) and producing (taking pictures, recording audio and video) rich media, coupled with anyplace anytime network access has proven to be a winning set of features that enabled mobile phones to gain such an extreme popularity.

Today we are witnessing two types of media convergence, both of which in a significant way are leveraged by mobile devices. Technical convergence implies that due to its digital nature, the same content may be available and accessible via different types of devices. For example, using a mobile phone or a PC, one can watch TV or read a newspaper online. Content convergence implies that similar or identical content is offered in different types of media and via different delivery channels. Content convergence is often used as a brand marketing strategy. For example, content related to the latest Indiana Jones sequel and its characters may be pushed via many media and non-media channels simultaneously, which may include themed web sites with video clips, still images, screen savers, wallpapers, and ringtones, as well as posters, toys, towels, bed sheets, and toys in Burger King happy meals. Both types of media convergence are most effectively aimed at young consumers and exploit their affinity to mobile devices. As a result, we can see that not only popular culture is influenced by consumer mobility, but also that popular culture itself helps reinforce and strengthen the role of mobile devices in the life of modern young people.

With the increase of technical capabilities of mobile communications, mobile phones have added a new virtual 
mobility dimension to the growing landscape of geographically dispersed social interaction [26]. Young people represent a significant portion of the mobile gadget consumers; many consider them a major driving force in the evolution and adoption of mobile technology [24]. To young people, mobile phone usage may go beyond the practical use of mobile phones used to make calls, texting, listening to music, or playing games. Many researchers emphasize the symbolic value of a mobile gadget, which may reflect the style and identity of a young person [18]. In the eyes of other young people, it may be very important to have a full address book on the "right" brand of a mobile phone and continuously receive a stream of text messages. Having grown up with a mobile phone, many modern college students may often find it as an item that helps determine their identity.

Young people, regardless of their nationality, cultural or ethnic background, seem equally interested in mobile games. This could be explained by "westernization" of the global society; Internet and mobile gadgets are viewed internationally by many young people as a "nice thing to have". A large international survey of young people in the US, Spain and Czech Republic [21] found that ubiquity of mobile devices had a greater effect on mobile game adoption than the perceived fun: young people clearly prefer the convenience to play a game at any time and any place using a small device that they carry around with them. Consistently across the countries, young people indicated that they prefer such a form of entertainment because it allows them to find something enjoyable to do when otherwise they would have been bored and forced to do nothing.

\section{What Do The StUdents SAY?}

To better understand the role of mobile culture and mobile devices in the life of contemporary students, in November and December of 2008 we surveyed a group 251 undergraduates from Central Connecticut State University, which included 117 females and 134 males, 25 years of age or younger, with an average age of 20.7 (Figure 1). This survey was offered to students who took a variety of general education courses; the survey instrument was designed to exclude a possibility for the same student to take it more than once. Not surprisingly, $100 \%$ of the survey respondents said that they have a mobile phone. CS majors comprised less than $3 \%$ of the survey respondents.

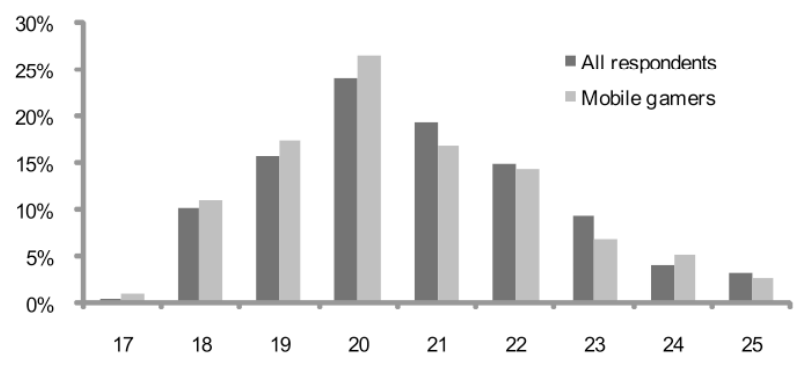

Figure 1. Age distribution of the survey respondents (in years)

Nearly the same percentage of males and females said that they regularly play mobile games: 66 (49\%) males and 55 $(47 \%)$ females. Both males and females in our survey said that they play mobile games on average 3.3 days a week. However, females tend to spend more time playing, as shown in Figure 2.

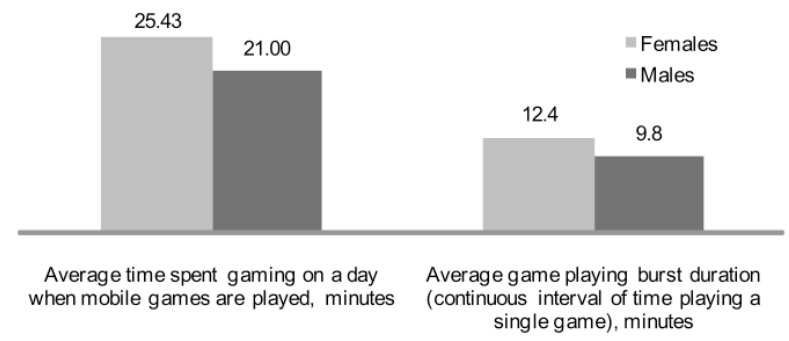

Figure 2. Time characteristics of mobile game playing

On average, students in this group started using a mobile phone when they were 15.4 years old, which is somewhat high. This reflects that the fact that a significant fraction of the respondents are first-generation college students, they come from working class families, and/or are in financial need. Therefore, giving a mobile phone to a child could have been considered a luxury in some of their families. Based on the data shown in Figure 3, it appears that the age when a person starts using a mobile phone influences whether they would play mobile games. For mobile gamers, the average age when they started using their first mobile phone was 14.8, while for nongamers it was 16.1 .

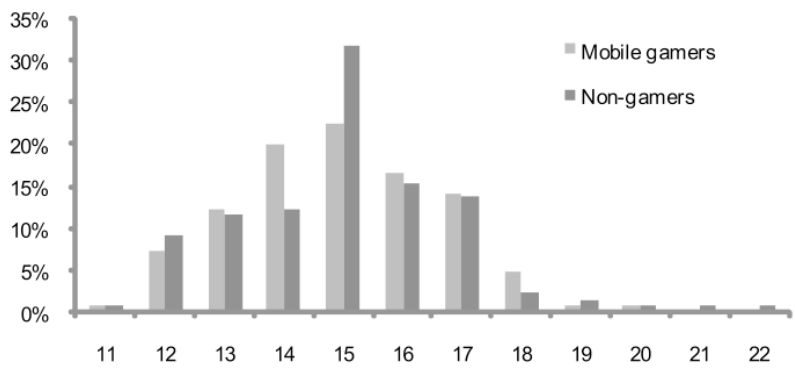

Figure 3. Distribution of age (in years) when respondents began using their mobile phones

Overall, surveyed mobile game players seemed more attached to and happier with their mobile phones - they were $23 \%$ less likely to plan a purchase of a new device. Furthermore, mobile gamers are using their mobile phones more actively than those who do not play mobile games: they make $6 \%$ more calls per day, send $22 \%$ more text messages per day, and keep $51 \%$ more songs on their mobile phones.

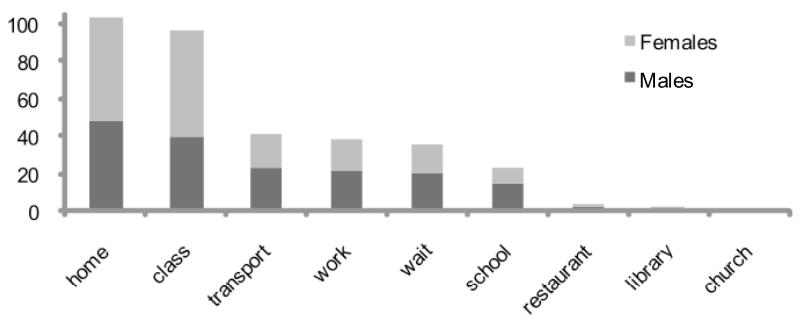

Figure 4. Most likely locations/situations and the number of survey respondents that play mobile games there

A number of studies of student attitudes towards computing (e.g. [8]) indicate that students choosing CS as a major tend to 
become interested in this field early in their life. Our findings are somewhat similar: the younger you are when you start using a mobile phone, the more interested you will be in using it in a wider variety of ways.

Our respondents were asked to name up to three different places where they are most likely to play a mobile game. A total of ten types of places were mentioned, all of which are shown in Figure 4. These included being at home with nothing else to do, in class during a lecture or another boring activity, in some form of transport (riding a bus or a train, at the airport or on a plane), at work (and apparently not working), while waiting for an appointment (e.g. at a doctor's office), at school between classes, and others.

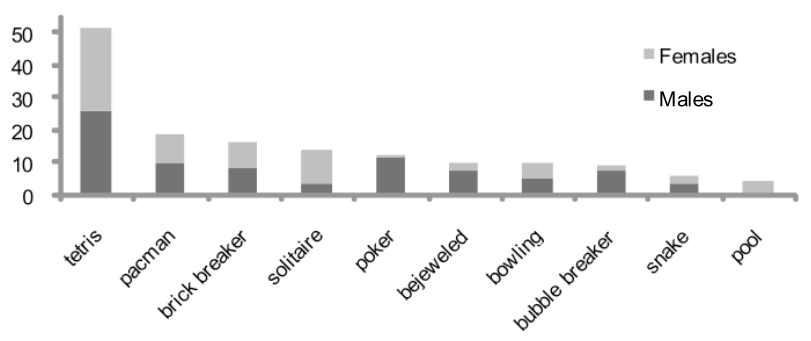

Figure 5. Top ten mobile games and the number of survey respondents that play them

In the survey, we asked to name up to three games that the responders play most frequently on their mobile phones. Out of 72 mobile games that were mentioned, Tetris, Pacman and Solitaire were the three most popular games, while over 40 other games were named only once. We did not make a distinction between different implementations of essentially identical games, such as different versions of Tetris. There is an approximately equal ratio between males and females who played the top ten games named by the respondents, which is especially clear for the top three games, as can be seen in Figure 5. Interestingly enough, Solitaire was played almost exclusively by females, while Poker was played almost exclusively by males. All of the top ten mobile games played by our respondents clearly belong to the class of casual games [15], which are characterized by a very simple gameplay. In fact, the genre and type of many game titles popular at arcades in the 80 's, today would be referred to as casual games due to their simplicity. Casual games appeal to "casual players" and are typically played in short bursts of time: while waiting in line, on public transportation, or, as our survey indicates, during a boring lecture. The rules of casual games are simple. Unlike many desktop or console games, casual games do not require any particular time commitment or any special skills. Most notably, many studies, including our survey, indicate that the demographics of mobile gamers are split equally between the two sexes $[6,14]$.

\section{How to Take Advantage of Mobile Games?}

Clearly, young people are very attached to their gadgets and use them on a daily basis. How can CS educators effectively take this sometimes disruptive technology and use it in a positive way?

\section{A. Mobile Computing}

ACM Computing Curriculum (CC) recognizes the role of mobile computing in today's world, as well as in the CS discipline. According to ACM CC, building interfaces for mobile systems is among many areas of practical capabilities in which prospective graduates should have experience, which also lists Mobile Computing as an elective course within the Net Centric Computing section of the body of knowledge. As a result, most of the very few CS departments that offer a course on mobile computing focus mostly on such topics as wireless network protocols, mobile data access, and perhaps mobile middleware. However, mobile computing can play a much more significant role as a motivational factor and as a learning context.

The importance of context has long been recognized in CS education [8,27]. Using a context allows educators to focus on a subject that is important and familiar to students while learning new concepts; it provides a motivational tool and offers a wider playing field for experimentation and engaging students in the educational process. Mobile computing and game development provide a relevant and a personally meaningful context, which allows students to use mobile gadgets that are near and dear to them. ACM CC mentions mobile computing as one possible learning context. As the data from IBM study [11] suggests that current students might be much more interested in using their mobile devices than PCs, the role of such a context becomes even more important.

A number of possible strategies for integrating mobile devices into computing curricula are presented in [19]. They range from using mobile devices as a programming platform in introductory courses to using them as a case study in an operating systems course. Current research shows that mobile devices and applications can serve as a valuable teaching and learning tool [5], in particular, to attract schoolchildren to computing [25], as a motivational context in computer literacy courses [19], and as an enabling technology used in augmented learning [13].

\section{B. Mobile Games}

Compared to traditional computer games, introduction of mobile game development into CS curricula received relatively little attention. Few examples include mobile game development courses within a graduate game development program [28] and a report on using mobile games as a motivating tool used to increase student interest in computing and other STEM disciplines [20]. Surprisingly, there are very few reports about leveraging the appeal and relative simplicity of mobile game development in order to spark and increase student interest in pursuing a degree in computing. Compared to desktop or console game development, mobile games are significantly less complex due to their simplified gameplay, smaller scale, simpler graphics, and other factors. Due to relative simplicity of many mobile games, many students with reasonable Java programming skills can develop a playable game within the timeframe of one semester. Additionally, mobile games can also be successfully used to broaden the horizons of students in introductory courses in CS by exposing them to a variety of advanced topics early in the curriculum. Therefore, it may be easier to adopt mobile game development 
in the CS curriculum than traditional game development, which is illustrated by the case study presented in this paper.

Traditional game development has been shown to be a good motivational tool, as reported in many publications on this topic, e.g. [17,22]. However, it is effective only for existing CS students who have already advanced down the course pipeline. In order to productively contribute to the development of a functional game, students must already have a substantial experience in software development. In contrast, development of fully functional mobile games could be offered even in introductory courses [16,19] and thus provide instant gratification to those students who may be just trying out a CS course to see if the entire program of study might fit their interest. Additionally, mobile computing can give the students another kind of instant gratification - they can quickly compile a working graphical application and play the resulting game on their own mobile device and proudly show it off to their friends. Our data indicates that nearly one half of all surveyed students play mobile games anyway, and we believe it would be wise to use this trend for the benefit of advancing CS education.

Current mobile applications range from custom interfaces of web-based social networking systems, online stores and banks, to unique mobile applications available exclusively on Apple App Store or Google Android Market. Students often have an easier time relating to and understanding the development of a smaller scale mobile application that they can test drive on their mobile device compared to sometimes nebulous realities of enterprise-scale software development. As a result, we have seen an increased student interest and motivation in studying CS and software development in the context of mobile computing. Not surprisingly, experience with mobile application development is often seen as a significant strength in today's job market and there is an increasing demand for such skills, especially in the area of mobile game development [2]. A recent report from In-Stat [12], a market research group, indicates that the current US mobile gaming market is worth over $\$ 1$ billion, while global mobile gaming revenues are expected to reach $\$ 6.3$ billion by 2013 . According to Gartner Research, mobile gaming industry is expected to grow at an annual rate of at least 10\% through 2011 .

Based on a survey of 1,200 representative US households [6], $36 \%$ of those surveyed reported that they play games on their mobile devices, up from $20 \%$ in 2002 . The same survey found that $40 \%$ of all game players were women. While the opponents of computer games in computing curriculum believe that women are not as interested in games as men [17], according to a number of studies, female students enjoy gamesoriented introductory computing courses more than male students $[10,17]$, and on average receive higher grades in such courses [4]. Our survey results show that not only an equal share of males and females play mobile games, but that females also spend more time playing games on their mobile phones.

\section{Mobile Development Platforms}

There are a number of software development platforms for mobile devices, which include Java Platform Micro Edition (Java ME), Adobe Flash Lite, Qualcomm's Binary Runtime
Environment for Wireless (BREW), Google's Android, Apple's iPhone SDK, etc. Each of these platforms has their unique advantages and disadvantages, but Java ME may be an all-around winner due to its cross-platform portability and wide popularity as a language of choice in many universities and Advanced Placement programs offered in US high schools.

Java ME (http://java.sun.com/javame/) provides a full set of tools for developing network-centric and interactive applications on mobile devices with various sets of hardware features. Sun Microsystems offers Java ME Software Development Kit (SDK) 3.0. Its previous version, Sun Java Wireless Toolkit (WTK) 2.5.2, can be used as a standalone tool or as a plug in with Eclipse Mobile Tools for Java (http://www.eclipse.org/dsdp/mtj/) or with NetBeans (http://www.netbeans.org/features/javame/). Regardless of which development environment is chosen, both Java ME SDK and WTK offer highly customizable emulators, which allow running and debugging of mobile applications without actually deploying them on the mobile device. These emulators allow developers tweak the settings such as screen size, amount of physical memory and processor characteristics to mimic the performance of an actual device.

\section{Challenges}

There are a number of challenges related to technology and curriculum that are associated with adopting mobile game development.

All mobile applications must conform to the unique constraints and features imposed by mobile hardware architectures. While processing power and amount of available memory has become less of a concern, there are a number of limitations that are here to stay. Few mobile devices have full keyboards and many applications have to rely on a numeric keypad for input. Displays are often very small and have varying sizes, which makes porting applications more difficult. Additionally, wireless environment imposes a number of limitations caused by intermittent and unreliable network connectivity with high latency. On the other hand, mobile devices often have unique hardware features not available on PCs, such as GPS sensors and accelerometers, which have been successfully used to empower a broad range of new mobile applications.

Maintaining the balance between educational and motivational aspects is a significant challenge to adoption of a new element in the computing curricula [23], whether it is robotics or game development. Critics indicate that we should not focus on computer games just because students like them [10]. Intrinsic complexity of developing a well-designed game is another considerable barrier to integrating games into the curriculum [3].

In our experience (described in detail in Section 6), mobile game development is uniquely positioned to address all of the challenges mentioned above. Because of a relative simplicity and a smaller scale, CS freshmen usually can develop a playable mobile game within a reasonable time - well within a single semester, even with limited programming skills. Many of the challenges mentioned above could be turned into advantages in terms of using them in the CS education process. 
Challenges of developing user interfaces for mobile devices can be a good topic for an in-depth discussion in a humancomputer interactions course, overcoming connectivity and security issues could provide a good study framework in a computer networks course, while mobile-specific development issues are a good topic for a software engineering course. Furthermore, mobile games can help broaden the horizons of many students in introductory computing courses by exposing them to a variety of advanced topics early in the curriculum [16].

It is important to remember, however, that whenever a particular innovation is integrated into a business process, it is necessary to ensure that the benefits outweigh the costs. Incorporating mobile computing into one or several CS courses or even an entire curriculum [19] is no exception and there must be a number of assessment mechanisms in place to evaluate the resulting effect.

\section{CASE STUDY: \\ A Freshman Mobile Game DeVElopment Course}

Here we describe an approach to teaching a Mobile Game Development course that is positioned early in the CS curriculum. The only required prerequisite is CS1: students must have some programming experience in Java. This course is based on a semester-long project to develop a playable game supplemented with an assortment of smaller game-centric assignments. Given a highly creative nature of this course, students were very strongly encouraged to discuss the design of their games, show off their work in progress, and brainstorm possible solutions with each other; however, they were required to do any programming work individually. Although we offer this course to all students meeting the prerequisite, it is ideally suited for freshmen with Advanced Placement (AP) credit, for whom it will be their first college-level CS course. The main objectives of this course are to introduce students to game development on mobile devices and expose them to various areas of CS that students are likely to encounter in upper-level courses, such as computer networking, artificial intelligence, databases, and computer graphics. An overarching goal of the course was to improve student motivation and engage them in studying CS since for many students this was their first course in the discipline.

The following sections emphasize the main topics discussed in our course. For the most part, they are presented here in the same sequence as in the course. However, different aspects of several topics, including data structures and software engineering, are discussed throughout the entire course.

\section{A. Programming Mobile Devices}

Students are first given an overview of mobile device architecture and a range of features that distinguish them from their desktop counterparts or gaming consoles. Students should have a clear understanding of the impact that small screens, limited CPU and battery power can have on the features of software that can work efficiently on these mobile devices. Very often students are surprised to learn that computational and storage capacity of many current mobile phones exceeds that of many decade-old desktop computers.
There are several software development platforms for mobile devices. Java ME is chosen here because Java is the primary language used in introductory college-level and Advance Placement CS courses. Java ME SDK described above offers all necessary tools for mobile software development with an emulator available on Windows and Linux platforms. Mobile game development is supported by several Java ME packages.

\section{B. Computer Graphics}

Since graphics is an integral part of any computer game, students are introduces to such elementary, but necessary prerequisites as graphics coordinate system and working graphics primitives. This mobile game development course is based on 2D graphics supported by several dedicated Java ME classes. We present fundamentals of computer animation from the perspective of traditional animation and moviemaking, stressing the concepts of frame rate, collision detection, and double buffering. Because mobile devices have relatively low processor speed and small screens, it is somewhat challenging to work with any advanced graphics. However, the context of this course offers a good opportunity to discuss such topics as digital sampling and image processing, alpha blending, raytracing, and $3 \mathrm{D}$ rendering, etc.

\section{HCI and Multimedia}

Students are introduced to the design principles and typical features of mobile games, most of which fall into the category of casual games. Interaction modalities used in computer games and mobile applications are very different from those found in other types of programs, especially those that students might have developed thus far in previous courses. Applications running on mobile devices must be built to accommodate a limited set of interaction options, which include small keypads and screens. Through discussions and experimentation, students are exposed to elementary research issues of human-computer interaction (HCI) when they see the impact of the limited attention span of a typical mobile user on the types of games they might enjoy.

Previous discussion of technical aspects of graphics and animation for mobile games serves as a bridge to a discussion of computer animation, music, and sound effects as building blocks used to create engaging mobile games. We offer a number of exercises that involve programming mobile devices to play tonal, WAVE and MIDI sounds and creating sound effects. In particular, students enjoy the exercise that involves developing a small piano keyboard on the screen of a mobile device that can be played using the numeric keypad.

\section{Data Structures and Algorithms}

Game screens and backgrounds are often created out of tiled images organized as 2D arrays, which offers an opportunity to discuss different types of data structures that students may encounter while creating a mobile game. For example, it is not uncommon for the users of slower mobile devices to press keys faster than they could be processed, leading to a discussion of queues. Different ways of moving from one screen to the next in a large game landscape could be used to introduce graphs. Naturally, this may lead to a 
discussion about different data structures and algorithms that are better suited for processing them. Although many of these data structures and algorithms are already implemented in Java $\mathrm{ME}$, students like to experiment with their own ideas. A game with multiple characters moving at the same time allows students to make their own choice of how and in which order to check for collisions among corresponding sprites and other objects present in the scene. This way, students get a firsthand experience seeing how an inefficient algorithm can ruin the gameplay leading to a discussion about algorithm efficiency.

\section{E. Artificial Intelligence}

Casual games, including those found on mobile devices, are typically designed to act by responding to the actions of a single player. For example, in Bejeweled, the game logic removes the line of adjacent gems of the same color formed by the user, and then allows the gems above to fall down and fill the gaps. Other types of games may involve more sophisticated AI-controlled behaviors making it possible for the user to play against the device. AI techniques used in our Mobile Game Development course include rule-based character behavior (e.g. chasing/evading) and adversarial search in turn-based games such as checkers. In one of the course assignments, students implement several behavior strategies so that game-controlled characters would chase or evade the character controlled by the player. Several examples are shown in Figure 6.

\section{F. Database Management}

Many students like to show off the results of their work, especially if this work involves developing a game on a mobile phone. Having high scores stored persistently on a mobile device adds more bragging rights and has a chance to increase the competitive edge of the game. This serves as a motivation for students to learn the difference between persistent and volatile data and the corresponding methods of storage. Java ME provides elementary facilities for persistent data storage that implements a two-column table. Using basic operations, such as storing and retrieving a record from the table, students are introduced to the topics of database management, data representation, and information retrieval.

\section{G. Computer Networking}

The question of creating multiplayer games for mobile devices will inevitably come up, once students acquire enough skills. Although this situation serves as a good point to discuss computer networking, the results of related hands-on activities were mixed. This was partially due to time limitations since this was one of the last topics in the course schedule. As a result, there may not be enough time to implement a new multiplayer game from scratch, or add multiple player capabilities to an existing project. Another reason for such an outcome was that compared to other topics presented in this course, implementation of data communication in Java ME demands relatively high technical skills that may be beyond the reach of students in this course. However, our students did have enough time and technical skills to experiment with the existing code for client/server message exchange implemented for mobile devices.

\section{H. Software Engineering}

Design and development of computer games is a good framework for teaching software engineering [6]. However, students need to be introduced to the basic rules and principles of software engineering throughout the entire CS curriculum. We followed this practice in this course, which is based on a semester-long project. Throughout the duration of the course, we gently introduce principles of software quality, present many examples of refactoring, and stress code optimization that plays an increasingly important role on mobile devices.

\section{I. $\quad$ Student Feedback}

This course has been offered to undergraduate students in the Spring 2009 semester. Although this course framework is expected to evolve, the unifying theme will stay the same: to engage students early in the CS curriculum through highly motivating applications using mobile devices.
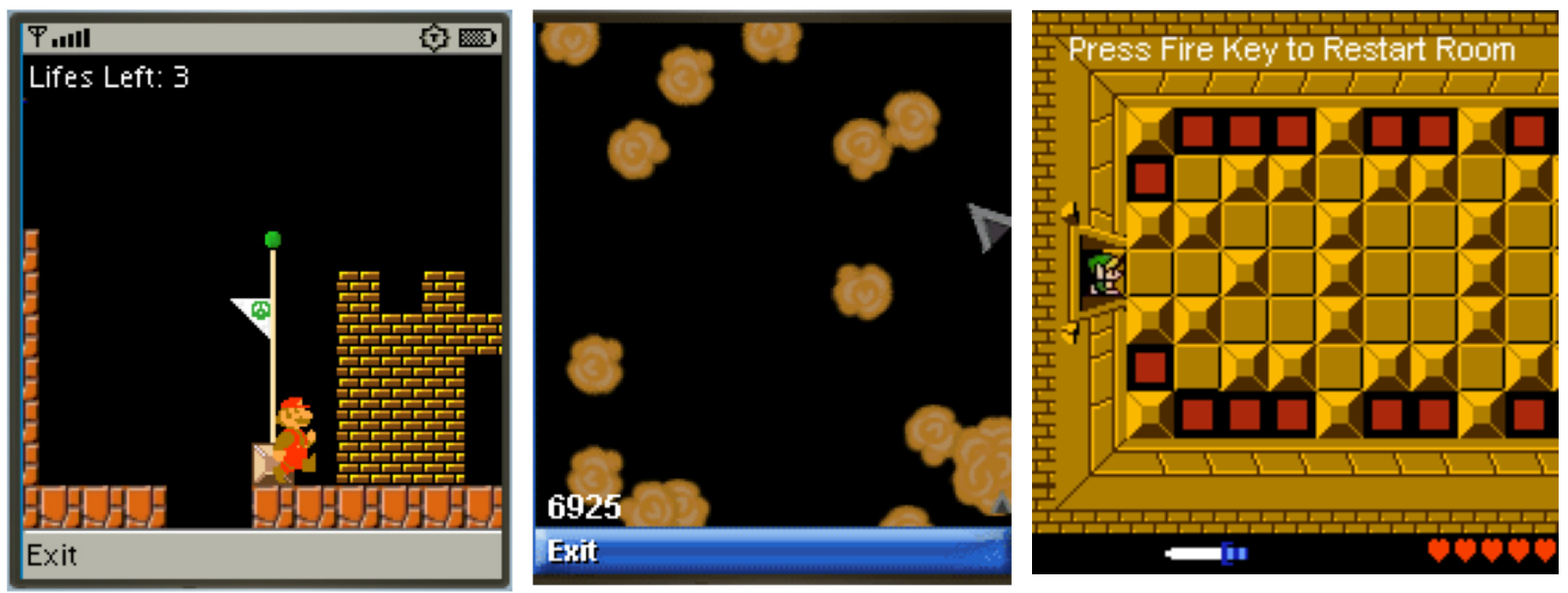

Figure 6. Mobile games created by students in the Mobile Game Development course: Mini Mario (left), Asteroids (middle), Mini Legend of Zelda (right) 
To assess the course outcomes, a survey of student opinions was administered at the beginning and at the conclusion of the course. Both times students were asked to use the scale of 1 to 10 to rate the degree to which different areas of CS are relevant to a successful career in computing. Each time, students were also asked about their plans to take a course in the corresponding area. Figure 7 summarizes the change in the student opinions between the start and the end of the course. It shows, for example, that at the end of the course students rated Artificial Intelligence as $26 \%$ more relevant to their careers than they did at the start of the course, while the number of students who planned to take a course in that area increased by $14 \%$ during the same period. It is obvious from these results that the course produced the desired outcome of improving the motivation and increasing student interest in pursuing a degree in CS.

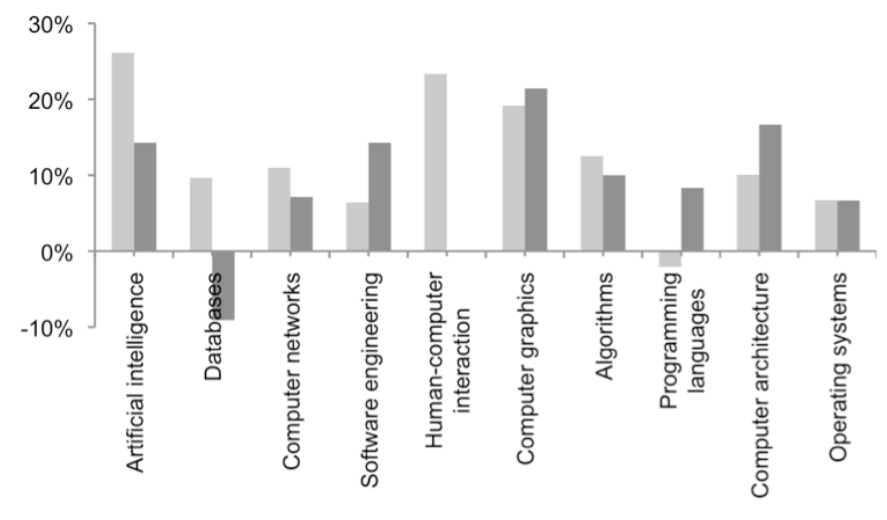

Relevance to career in CS, \% change =Planning to take a course, $\%$ change

Figure 7. Change in student opinions about CS after taking a Mobile Game Development course

One of our goals was to convince the students who decided to give CS a try was that "it's not all about coding" and that it includes many other diverse areas. Many students agreed:

- The course definitely sparked my interest in [other areas] and made me want to explore.

- It gave me more experience and depth of knowledge in certain areas.

Students liked the fact that in this course they were exposed to several advanced topics. Most importantly, this motivated some students to learn more about them:

- It did make me want to learn about databases, networking, etc. to broaden my understanding of CS. Not be limited to just the software side.

- I enjoyed learning about new topics such as AI. Our games [were] playable over the network, so now I am more interested in networking.

- I think this course [exposed] the software engineering part of CS. I think it gave me more of an idea as to how much work and time goes into making a game.

Many students appreciated the teamwork and the semesterlong project that resulted in creating playable mobile games (Figure 6):
- The project was the most fulfilling and enjoyable part of the course.

- It's always good to be exposed to group work -- few projects in the real world are done by a single individual or no input from others. And we all know that us CS folk could always use some more practice dealing with others.

- I liked how everyone got to make their own game.

\section{CONCLUSION}

As many young people are contemplating making a switch in their usage habits from PCs to mobile devices, mobile gaming becomes an increasingly more popular activity. Due to the ubiquity of mobile devices, most mobile games can be enjoyed anytime and anyplace. Furthermore, unlike many PCand console-based games, mobile games usually do not require any special skills and can be played in shorter periods of time.

Mobile computing has been recommended by the ACM Computing Curricula as one of the contexts to embed teaching of computing concepts. Mobile game development can serve as an exciting learning context that is both motivational and technically challenging. Although many critics say that computer games is almost exclusively a males' domain and that they promote violence, many studies including our own survey indicate that most people play casual games (puzzles, etc.) on their mobile phones, an equal percentage of females and males enjoy mobile games, and females spend more times doing so.

In our approach to integrating mobile game development into CS curriculum, we strive to make connections to advanced topics as early in the program as possible. Many studies indicate that students often get disillusioned in CS because they see too many irrelevant or contrived examples that are boring or have very little to with real-world applications. There is a certain percentage of students who are interested in other areas of CS that emphasize design or theory over coding. By exposing students to a wide range of advanced topics early in their academic career, our approach aims to show students that CS can be much more exciting than coding and that there are so many areas in which programming plays a supplementary role. Course structure described in this paper presents a cohesive and engaging theme for introducing many advanced topics early in the CS curriculum.

As our survey shows, whether we like it or not, our students do play games on their mobile phones and mobile gaming is here to stay. It is up to us, however, to use this situation for the benefit of CS education and turn it from a disruptive technology to a motivational tool.

\section{REFERENCES}

[1] Beaubouef, T. and Mason, J. 2005. Why the high attrition rate for computer science students: some thoughts and observations. SIGCSE Bull. 37, 2 (June 2005), 103-106.

[2] Brandel, M. 2007. 12 IT skills employers cannot say no to. ComputerWorld, Jul. 2007.

[3] Burd, B.et al. 2007. Computer games in the classroom, or, how to get perfect attendance, even at 8 AM. SIGCSE Bull. 39, 1 (Mar. 2007), 496496. 
[4] Chamillard, A. T. 2006. Introductory game creation: no programming required. SIGCSE Bull. 38, 1 (Mar. 2006), 515-519.

[5] Chao, C. 2006. An investigation of learning style differences and attitudes toward digital game-based learning among mobile users. In Proc. $4^{\text {th }}$ IEEE International Workshop on Wireless, Mobile and Ubiquitous Technology in Education (Nov. 16-17, 2006), 29-31.

[6] Claypool, K. and Claypool, M. 2005. Teaching software engineering through game design. SIGCSE Bull. 37, 3 (Sep. 2005), 123-127.

[7] Entertainment Software Association. 2008. Essential facts about the computer and video game industry. $\mathrm{http} / / / \mathrm{www}$. theesa.com/facts/pdfs/ESA_EF_2008.pdf.

[8] Forte, A., Guzdial, M. 2004. Computers for communication, not calculation: media as a motivation and context for learning. In Proc. $37^{\text {th }}$ Hawaiian International Conference of Systems Sciences (Big Island, HI Jan. 5-8, 2004).

[9] Goggin, G. 2008. Mobile Phone Culture. London. Routledge.

[10] Haller, S. et al. 2008. Games: good/evil. SIGCSE Bull. 40, 1 (Feb. 2008), 219-220.

[11] IBM Study Finds Consumers Prefer a Mobile Device Over the PC. 2008. http://www-03.ibm.com/press/us/en/ pressrelease/25737.wss

[12] In-Stat, 2008. Mobile Applications Series: US Mobile Gaming 20082013. Wcatalogue.asp?id=231\#IN0804316MCM

[13] Klopfer, E. 2008. Augmented Learning: Research and Design of Mobile Educational Games. MIT Press.

[14] Koivisto, E. M. 2006. Mobile games 2010. In Proc. 2006 international Conference on Game Research and Development (Perth, Australia, Dec. 4-6, 2006), vol. 223, 1-2.

[15] Kuittinen, J. et al. 2007. Casual games discussion. In Proc. 2007 conference on Future Play. (Toronto, Canada, Nov. 14-17, 2007), 105112.

[16] Kurkovsky, S. 2009. Engaging students through mobile game development. In Proc. $40^{\text {th }}$ ACM Technical Symposium on Computer Science Education (Chattanooga, TN, Mar. 4-7, 2009).
[17] Leutenegger, S. and Edgington, J. 2007. A games first approach to teaching introductory programming. SIGCSE Bull. 39, 1 (Mar. 2007), 115-118.

[18] Ling, R. 2004. The Mobile Connection. The Cell Phone's Impact on Society. Morgan Kaufmann.

[19] Mahmoud, Q. 2008. Integrating mobile devices into the computer science curriculum. In Proc. $38^{\text {th }}$ ASEE/IEEE Frontiers in Education Conference (Oct. 22-25 2008), S3E:17-25.

[20] Metcalf, D. et al. 2008. My sports pulse: increasing student interest in STEM disciplines through sports themes, games and mobile technologies. In Proc. $5^{\text {th }}$ IEEE International Conference on Wireless, Mobile, and Ubiquitous Technology in Education (Beijing, China, Mar. 23-26, 2008), 23-30.

[21] Okazaki, S., Skapa, R., Grande, I. 2008. Capturing global youth: mobile gaming in the U.S., Spain, and the Czech Republic. Computer-Mediated Communication, 13 (2008), 827-855.

[22] Overmars, M.H. 2004. Learning object-oriented design by creating games. IEEE Potentials, 23, 5 (Dec. 2004), pp. 11-13.

[23] Repenning, A. and Ioannidou, A. 2008. Broadening participation through scalable game design. SIGCSE Bull. 40, 1 (Feb. 2008), 305-309.

[24] Sarker, S., Wells, J.D. 2003. Understanding mobile handheld device use and adoption. Comm. ACM, 46, 12 (Dec. 2003), 35-40.

[25] Sharples, M. 2000. The design of personal mobile technologies for lifelong learning. Comput. Educ. 34, 3-4 (Apr. 2000), 177-193.

[26] Thulin, E., Vilhelmson, B. 2007. Mobiles everywhere: youth, the mobile phone, and changes in everyday practice. Tidskriftforeningen Young 15 (2007), 235-253.

[27] Xu, D., Blank, S., Kumar, K. 2008. Games, robots, and robot games: complementary contexts for introductory computing education. In Proceedings of the $3^{\text {rd }}$ International Conference on Game Development in Computer Science Education, (Miami, FL, Feb. 27 - Mar. 03, 2008) 66-70.

[28] Zyda, M. J., Thukral, D. Ferrans, J. 2008. Incorporating mobile games into a computer science game degree program. In Proc. Microsoft Academic Days Conference on Game Development in Computer Science, (Celebrity Century, Feb. 28 - Mar. 2, 2008). 